\title{
Understanding DC-Bias Sputtered Thorium Oxide Thin Films Useful in EUV Optics
}

\author{
William R. Evans \\ Sarah C. Barton \\ Michael Clemens \\ David D. Allred \\ allred@byu.edu
}

Follow this and additional works at: https://scholarsarchive.byu.edu/facpub

Part of the Astrophysics and Astronomy Commons, and the Physics Commons

\section{Original Publication Citation}

William R. Evans, Sarah C. Barton, Michael Clemens and David D. Allred, "Understanding DC-Bias Sputtered Thorium Oxide Thin Films Useful in EUV Optics [6317-37]," in: Advances In X-Ray/EUV Optics, Components, And Applications, edited by Ali M. Khounsary, and Christian Morawe, Proceedings of SPIE, 6317, 631711-1 to 8 (26). [http://spiedigitallibrary.org/proceedings/ resource/2/psisdg/6317/1/631711_1?isAuthorized=no][http://dx.doi.org/1.1117/12.687499].

\section{BYU ScholarsArchive Citation}

Evans, William R.; Barton, Sarah C.; Clemens, Michael; and Allred, David D., "Understanding DC-Bias Sputtered Thorium Oxide Thin Films Useful in EUV Optics" (2006). Faculty Publications. 990. https://scholarsarchive.byu.edu/facpub/990 accepted for inclusion in Faculty Publications by an authorized administrator of BYU ScholarsArchive. For more information, please contact ellen_amatangelo@byu.edu. 


\title{
UNDERSTANDING DC-BIAS SPUTTERED THORIUM OXIDE THIN FILMS USEFUL IN EUV OPTICS
}

\author{
William R. Evans, Sarah C. Barton, Michael Clemens and David D. Allred* \\ Brigham Young University, Provo, UT
}

\begin{abstract}
We used spectroscopic ellipsometry to determine the optical constants of seven thin-film $\mathrm{ThO}_{2}$ samples deposited by radio-frequency sputtering, thickness ranging between 24 and $578 \mathrm{~nm}$, for the spectral range of 1.2 to 6.5 . We used a hollow-cathode light source and vacuum monochromator to measure constants at $10.2 \mathrm{eV}$. None of the deposition parameters studied including DC-bias voltages successfully increase the $n$ of (that is, densify) thoria films. ${ }^{1,2}$ The value of $n$ at $3.0 \mathrm{eV}$ is $1.86 \pm 0.04$. We find compelling evidence to conclude that the direct band gap is at $\sim 5.9 \mathrm{eV}$, clarifying the results of others, some of whom observed the absorption edge below $4 \mathrm{eV}$. The edge in the two thickest films is of a narrow feature $(\mathrm{FWHM}=0.4 \mathrm{eV})$ with modest absorption $\left(\alpha \sim 6 \mu \mathrm{m}^{-1}, k \sim 0.1\right)$. Absorption may go down briefly with increasing energy (from 6.2 to $6.5 \mathrm{eV}$ ). But at $10.2 \mathrm{eV}$ absorption is very high and index low as measured by variableangle reflectometry, $\alpha=47.3 \pm 5.5 \mu \mathrm{m}^{-1}$ and $k=0.48 \pm 0.05$, and $n=0.87 \pm 0.12$.
\end{abstract}

Keywords: Band gap, optical constant, thickness measurement, thorium oxide, spectroscopic ellipsometry, thoria, refractive index; DC-bias, sputtering, x-ray diffraction

\section{INTRODUCTION}

Determining the optical properties, particularly the band gap and refractive index, of technological materials such as thorium dioxide (thoria $=\mathrm{ThO}_{2}$ ) is important for basic science as well as for technological reasons. Learning how to densify thin films is critical for using it as an EUV material since front surface near-normal reflectance goes as relative density squared. Thoria is the most refractory oxide known with a melting temperature is above $3500 \mathrm{~K}$. It, along with the important nuclear oxides: $\mathrm{UO}_{2}$ and $\mathrm{PuO}_{2}$, and the nonnuclear $\mathrm{CeO}_{2}$, constitute the binary oxides with the fluorite structure.

However, thoria is the only one of the four whose oxide has a stable +4 oxidation state. That is, it can be depended to be nearly perfectly stoichiometric. This makes it important for optical studies and applications. It has higher visible and near UV optical transparency than the other fluorite oxides and a number of its applications, including optical coatings ${ }^{3}$, and candoluminescence, are based on this fact. It is the primary material in Welsbach gas mantles which are widely used in portable camping lanterns. There is also an interest in understanding the optical properties of the oxides and fluorides of heavier group IV metals related to their capacity to act as hosts to rare earth and transition metal ions. These have applications as gamma radiation detectors and phosphors. This has led to many experimental ${ }^{4}$ and some computational studies. ${ }^{5}$ Since $\mathrm{ThO}_{2}$ is the only stable oxide of thorium and its $\Delta \mathrm{G}<<0$, the surface of thorium metal and many thorium alloys in nonreducing ambients can be expected to be coated with thoria. This will influence the apparent optical properties of the metal.

Considering how important thorium and thoria are and how much they have been studied in the past half century, it is surprising that the optical constants of thoria are not better known. In fact, there has been no general agreement on the band gap of thoria; some studies placing the absorption edge near or below $4 \mathrm{eV} .{ }^{6}$ One of the purposes of this report is to show that the direct band gap of thoria is much larger than 4 . It is at $\sim 5.9 \mathrm{eV}$, confirming the proposals made earlier by some researchers (observing a few very pure bulk crystals) that the fundamental absorption edge occurs at or above $5.75 \mathrm{eV} .^{7,8} \mathrm{We}$ will also present evidence that this edge corresponds to a narrow absorption band followed by a region of less absorption in the case of our two thickest films. We suggest that this narrow absorption feature is possibly related

allred@byu.edu; phone: (801) 422-3489; www.physics.byu.edu/faculty/allred/cv/CV.htm 
to the empty $5 \mathrm{f}$ orbitals ${ }^{5}$ of $\mathrm{Th}$ in $\mathrm{ThO}_{2}$. The absorption edge may be quite different in character than in covalently bonded semiconductors like Si. Further work was conducted to see if the lowest sp to sp-type transitions characteristic of familiar semiconductors (like silicon, diamond, etc.) may lie in the VUV. One contribution of this study then is to extend the knowledge of thoria's optical constants into the VUV.

In addition to the above mentioned applications, thin-film thorium and thoria have been recently investigated as a reflector for the extreme ultraviolet (EUV) and soft x-rays. Its low angle reflectance is twice as high as standard materials at about $200 \mathrm{eV} .{ }^{9,10}$ Progress in this area is limited, however, by a lack of knowledge of the properties of the deposited materials including film thickness and density. An understanding of factors that can affect the characteristics of the thin film during deposition is central to this knowledge. In a previous publication, ${ }^{1}$ we reported that DC-bias voltage has no effect on the index of refraction for reactively sputtered thoria thin films. Bias voltage has been related to achieving higher density films.

If the surface of a porous material is densified it will have higher EUV reflectances. Consider normal-incidence reflectance of a thick film. This is equal to $1 / 4\left[\delta^{2}+\beta^{2}\right]$. ( $\beta$ is also termed $k$ ), Since $\delta$ and $\beta$ are proportional to density, the normal-incidence reflectance of a film which is only $70 \%$ dense $^{\dagger}$ will only be about $50 \%$ that of a $100 \%$ dense material. The increase in reflectance with densification is less at angles closer to grazing, but techniques which produce films closer to $100 \%$ dense should be sought out to produce surfaces and multilayers with the highest reflectances.

We did not set out to measure absorption nor to determine the band gap of thoria, but the use of spectroscopic ellipsometry to obtain film thickness and index led directly to an examination of band gap. The ellipsometer necessarily provides absorption data since fully modeled data will provide thickness and $n$, and $k$ at each energy. The imaginary part of the complex index of refraction, $k$ is related to absorption by $\alpha=4 \pi k / \lambda$. There is even less reason to expect a strong effect on the band edge with bias. Bombardment might be expected to produce states in the forbidden gap but the direct gap band edges are characterized by the sum of all transitions and are not strongly changed with a low density of point defects.

\section{EXPERIMENTAL}

Thin-film thorium oxide samples were deposited via reactive, radio-frequency (RF), magnetron sputtering using a US Inc. Mighty Mak 4-inch gun, powered with a Plasmatherm $3 \mathrm{~kW}$ RF power supply. More details are available in references $1 \& 2$. The incident power was set to about $300 \mathrm{~W}$ and there was about $20 \mathrm{~W}$ of reflected power once the plasma lit. The base pressure of the turbopumped (4 inch) and cryopumped (CryoTorr 8 ) Cu-gasketed, high vacuum chamber was less than about $1 \times 10^{-2} \mathrm{~Pa}(0.1 \mathrm{mtorr})$. Both high vacuum pumps were used during the depositions. The gate valve in front of the cryopump was manually set to be almost completely closed. The turbo pump was not throttled. The working gas was a mixture of argon and about $20 \%$ oxygen. The total pressure was normally at about 1.1-1.3 Pa (8-10 mtorr). The oxygen flow was set via a sapphire leak valve to about $0.33 \mathrm{~Pa}$ ( $2.5 \mathrm{mtorr})$ using the system's ion gauge before the $\operatorname{Ar}(99.999 \%)$ was introduced. Under these conditions the normal deposition rate was about $1 \mathrm{~nm} / \mathrm{min}$. A 101-mm diameter, $6.5-\mathrm{mm}$ thick thorium target, previously cut from a cast thorium ingot, was used in a sputter-up geometry. The target-to-substrate distance was about $0.25 \mathrm{~m}$. A moveable shutter lay $0.055 \mathrm{~cm}$ below the substrate.

The deposition substrates were silicon wafer pieces from standard polished silicon wafers (100 orientation) and pieces broken from synthetic, fused quartz slides (G. Finkenbeiner, Inc. 781 899-3138) chosen to possess high UV transmission at $6.5 \mathrm{eV}$. Atomic Force Microscopy (AFM) measurements have shown the typical rms roughness of similar wafers to be $\sim 0.2 \mathrm{~nm}$ over a $100 \mathrm{~nm} \times 100 \mathrm{~nm}$ area. The thickness of the native silicon dioxide of the silicon wafers used was estimated to be $2.0 \mathrm{~nm}$, which we found to be the average for similar wafers.

The samples were suspended upside-down above the sputter gun. The sample holder was attached to a $9.5 \mathrm{~mm} \mathrm{Cu}$-post (Ceramaseal ${ }^{\mathrm{TM}}$ ) feed through (on a $23 / 4$-inch conflat). This allowed the substrates to be connected to a negative bias

${ }^{\dagger}$ We found evidence that thin film thoria samples prepared by a variety of techniques may be only $\sim 70 \%$ dense. $^{1}$ 
voltage and be isolated from the rest of the chamber. Some of the samples were sputtered at negative DC bias voltages with a magnitude of up to $68 \mathrm{~V}$.

\section{CHARACTERIZATION:}

The deposition system had a quartz-crystal monitor positioned to see most of the flux which struck the substrates and was not blocked by the shutter which protected the sample holder until the sputter rates were stabilized. This allowed us to achieve the approximate film thickness desired. We obtained a more accurate measurement of each film's thickness using low-angle x-ray thin-film interference. Following each deposition we measured the low-angle $\left(\sim 0.6^{\circ}-1.8^{\circ}(2 \theta)\right)$ $\mathrm{x}$-ray reflection (XRR) spectrum of the reflectance sample, using a Scintag ${ }^{\mathbb{R}}$ model XDS 2000 X-ray Diffractometer, with $\mathrm{Cu}-\mathrm{K}_{\alpha}$ radiation $(0.154 \mathrm{~nm})$. To determine the thickness of the $\mathrm{ThO}_{2}$ layers we compared the observed position of interference minima in the measured XRR spectrum with those modeled for a range of Th thicknesses on $2 \mathrm{~nm}$ of $\mathrm{SiO}_{2}$ (typical thickness of native oxide) on Si substrates. Bissell et al discuss this process in more detail. ${ }^{11}$

We also measured the $x$-ray scattering of the two thickest films over the range $15^{\circ}$ to $90^{\circ}(2 \theta)$ and determined that the films were polycrystalline thoria with a preferred (111) and (110) orientations. The (100) and similar low-angle orientations are reduced. Table 1 summarized the XRD data and our interpretation. It can be noted that a lack of crystallites with (100) planes parallel to the film's top surface is also seen in atomically deposited materials with the diamond cubic structure. The diamond cubic and the fluorite structures are quite similar. In the diamond cubic structure half of the tetrahedral vacancies in the parent face-centered-cubic structure are filled, while in the fluorite structure all are filled. The account for why the thin film diamond cubic materials like silicon eschew 100 surfaces is based on energetic arguments. The (111) and the (110) have the maximum density of atoms on their growing surfaces and the minimum number of dangling bonds. The minimum surface energy is obtained when the maximum bonds are formed and the least unstable dangling bonds are seen. This same argument could be used with $\mathrm{ThO}_{2}$ with its fluorite structure though attention should be paid to the partially ionic nature of the material.

Table I shows the relative intensities (background removed) of scattered $\mathrm{Cu} \mathrm{K}_{\alpha}$ X-rays at each angle between $15^{\circ}$ and $87^{\circ}$ (20) by XRD from sample ThO2 050604-2 (thickness $=357 \mathrm{~nm}$ ). The first column of values provides the peak

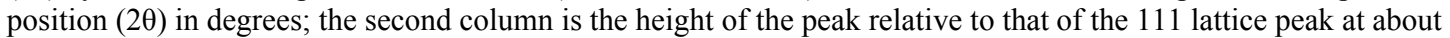
$27.4^{\circ}$, with the middle entry being the number indexing the lattice planes. The fourth and fifth columns of values show the standard positions and relative intensities of the peaks for $\mathrm{ThO}_{2}$, as provided by the Powder Diffraction File. ${ }^{12}$

\begin{tabular}{|ccccc|}
\hline Location & Relative Size & $\begin{array}{c}\text { Crystal } \\
\text { Orientation }\end{array}$ & $\begin{array}{c}\text { Expected } \\
\text { Position }\end{array}$ & $\begin{array}{c}\text { Expected } \\
\text { Intensity }\end{array}$ \\
\hline 3.253 & $100 \%$ & $(111)$ & 3.234 & $100 \%$ \\
\hline 2.822 & $13.34 \%$ & $(200)$ & 2.800 & $35 \%$ \\
\hline 1.996 & $64.73 \%$ & $(220)$ & 1.980 & $58 \%$ \\
\hline 1.705 & $29.00 \%$ & $(311)$ & 1.689 & $64 \%$ \\
& & $(222)$ & 1.616 & $11 \%$ \\
& & $(400)$ & 1.400 & $8 \%$ \\
\hline 1.281 & $11.82 \%$ & $(331)$ & 1.284 & $26 \%$ \\
& & $(420)$ & 1.252 & $17 \%$ \\
& & $(422)$ & 1.143 & $20 \%$ \\
& & $(511)$ & 1.108 & $19 \%$ \\
\hline
\end{tabular}

The composition of one of the samples was also measured using x-ray photoelectron spectroscopy (XPS) giving a Th to $\mathrm{O}$ ratio of approximately 1 to 2 throughout the thickness of the sample, as we would expect for $\mathrm{ThO}_{2}{ }^{1,2}$

The surface roughnesses of our films were measured using a Veeco Instruments Dimension 3100 Atomic Force Microscope at Brigham Young University. The surface roughness, measured on a 1-micron by 1-micron area and averaged over two spots on each of four samples, was $5.1 \pm 0.4 \mathrm{~nm}$ and appeared to be independent of thickness. (However, N. F. Brimhall, in these proceedings measured roughness a factor of three less than these. ${ }^{10}$ ) This value for 
roughness was used in modeling the films' ellipsometric data. Figures 1a and $1 \mathrm{~b}$ show the surface topography from the films 050818 and 050604-2, which are 578 and $357 \mathrm{~nm}$ thick, respectively.
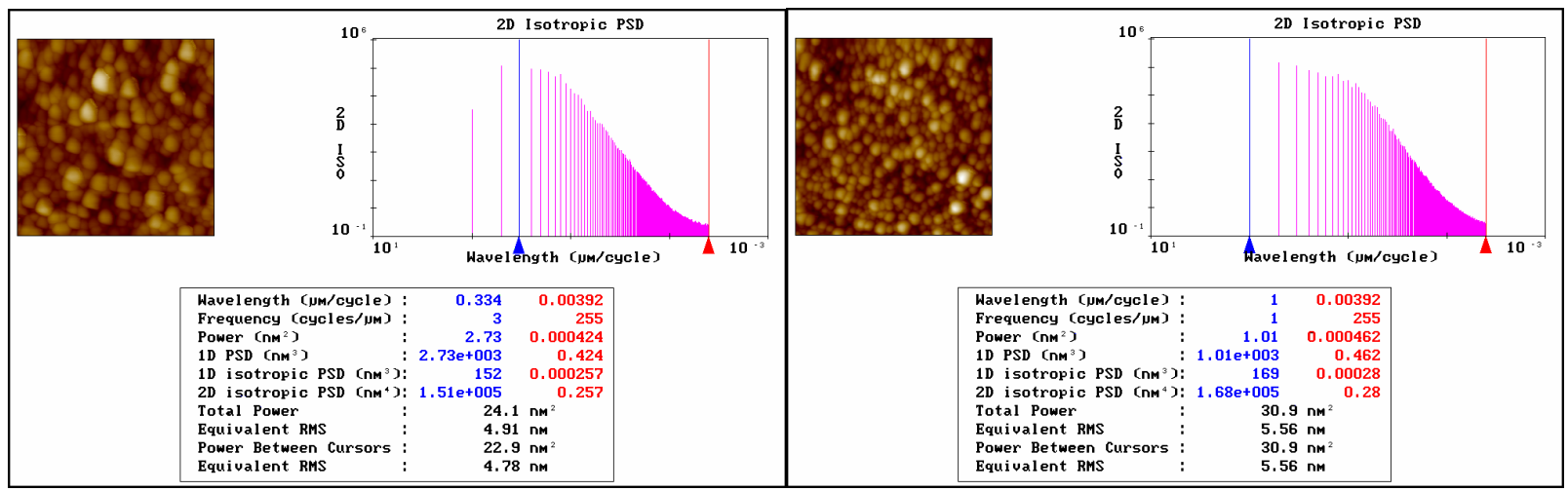

Figures 1a \& 1b: Showing the surface topography for samples ThO2 050818 on the left (a), and ThO2 050604-2 on the right (b). These samples were measured to have thicknesses of 578 and $357 \mathrm{~nm}$ and were sputtered at 65 and $0 \mathrm{~V}$, respectively.

\section{Optical Constants}

Optical properties of our thin films were measured between 1.24 and $6.5 \mathrm{eV}$ using a John A. Woollam Company M2000 Spectroscopic Ellipsometer at BYU. Ellipsometric reflectance data $(\Psi$ and $\Delta)$ was taken on the silicon samples at angles between $67^{\circ}$ and $83^{\circ}$ from normal, taking measurements every degree. Data were also taken at $10.2 \mathrm{eV}(121.6$ nm-H Lyman alpha) using a McPherson 225 Vacuum Monochromator at BYU. The monochromator uses a hollow cathode light source with $\mathrm{H} 2$ gas. Measurements were made with a Channeltron detector in high vacuum (about $10^{-6}$ torr) between 2.5 and 60 degrees from grazing. Typical count rates were $70 \mathrm{~K} / \mathrm{s}$ and the dark current is low $(<1 \mathrm{cps})$. Figure 1 is a schematic showing how such data were obtain.
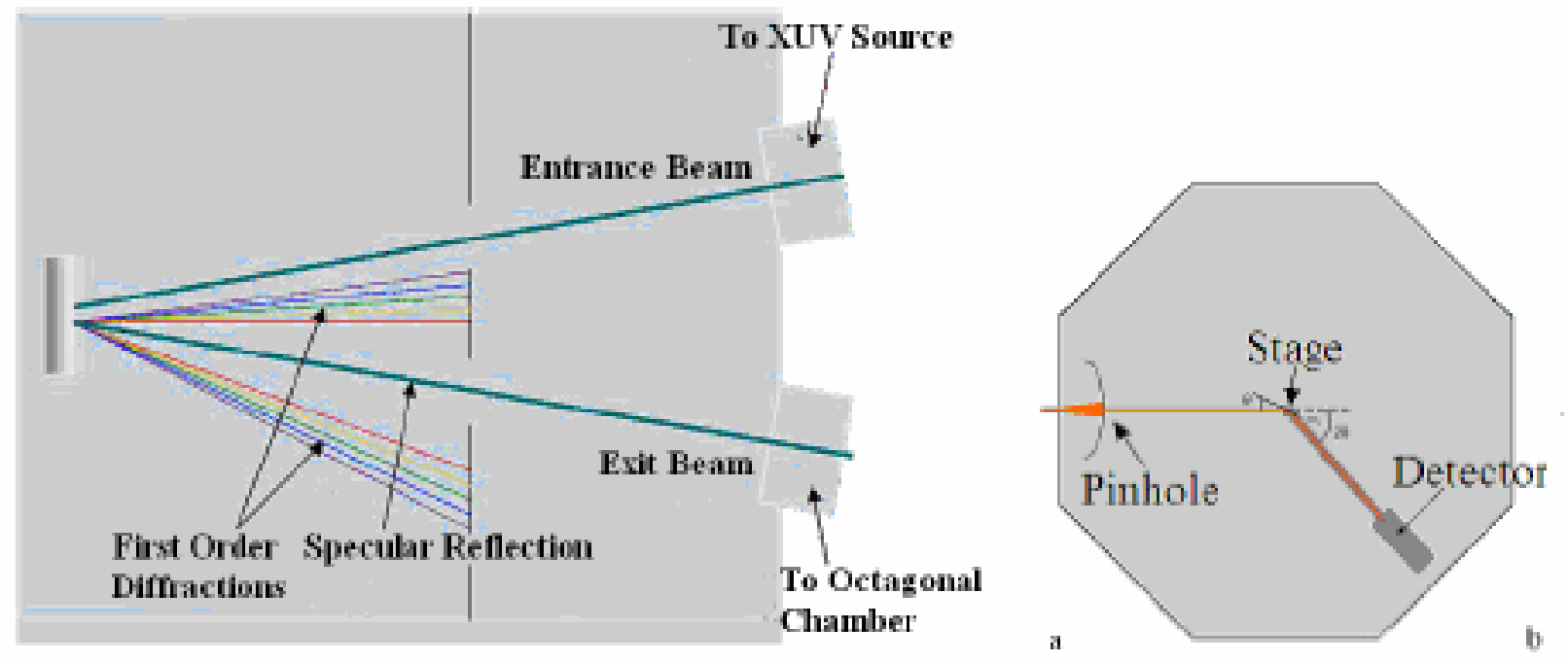

Figure 2: a) McPherson Vacuum monochromator b) reflection measurement in octagonal-chamber. Figure courtesy N. Brimhall (2006).

The ellipsometer at BYU is also set up to take normal-incidence transmission measurements. These are extremely important in constraining $k$. We took normal incidence transmission measurements over the same energy range on the 
samples deposited on quartz. Optical constants were then modeled using the WVASE software provided with the

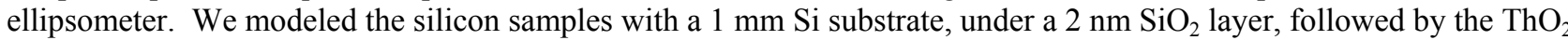
layer whose optical constants we were modeling. Then a layer of roughness, set at the root mean squared value determined by AFM. The thickness of the $\mathrm{ThO}_{2}$ layer was initially set at the value found by $\mathrm{XRD}$, but then we allowed it to vary somewhat. We used this method because fits done without allowing the thickness to change, were visibly and quantitatively (larger error term) poor. We were able to achieve a "mean square error" (roughly equivalent of $\chi^{2}$ ) of less than 2.5 for the modeled optical constants in all but the thickest samples. This means that the model fit the data close to ideally. The measurement is vastly over-determined, since the model has only between 9 to 11 parameters, whereas the thousands of points of data are collected for each sample. All thicknesses quoted are ellipsometric thicknesses.

\subsection{Index}

\section{REPORTED DATA}

We observed no correlation of $n$ with bias voltage, sputter pressure, deposition rate, or any other deposition parameter monitored. This is more fully discussed in ref. [1]. The non-dependence of $n$ on these parameters is best illustrated in Figure 3. Consider, for example, bias voltage. The average value of $n$ at $3 \mathrm{eV}$ was $1.86 \pm 0.04$ for both the unbiased, and the biased samples. Since there does not seem to be a significant correlation between the sputtering bias voltage, and the value of $n$, DC- bias voltage cannot be reliably expected to increase the index of refraction of our $\mathrm{ThO}_{2}$ thin films. However, substrate heating is currently being investigated. Sviridova and Suikovskaya's samples showed higher index after heating.3

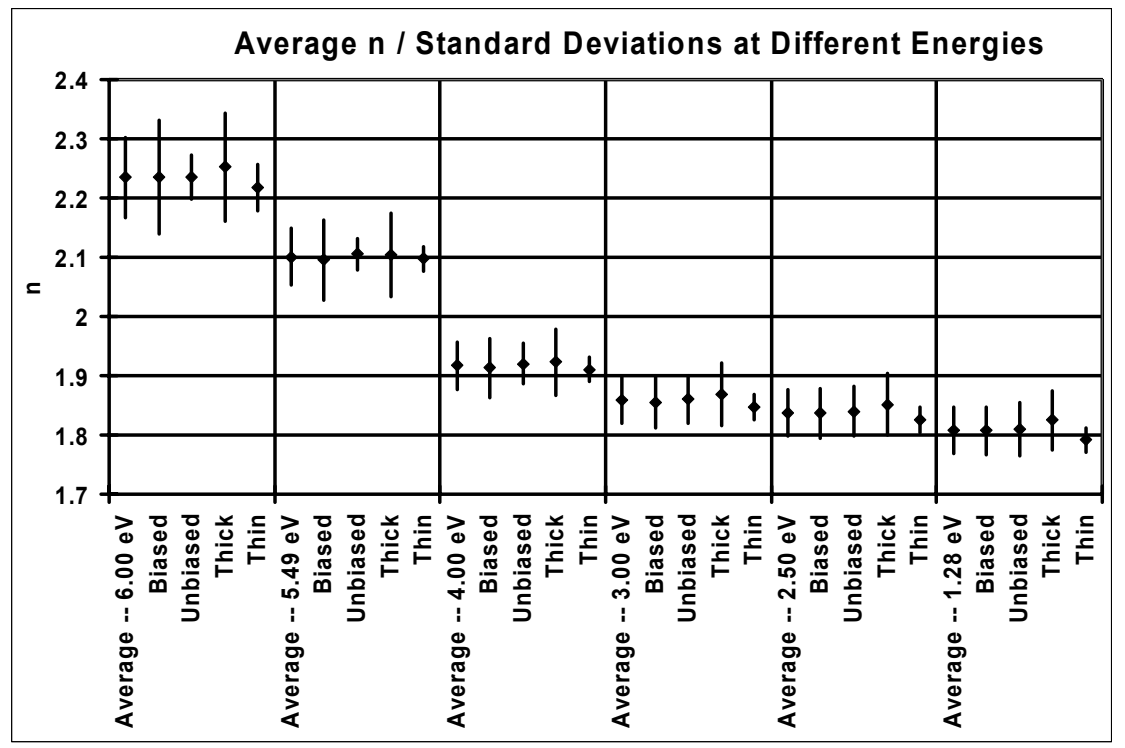

Figure 3: Showing average $n$ and standard deviations at different energies. Each major division represents a different energy. The first item in the division is the average for all the samples at that energy with the standard deviation shown as error bars. The second and third items are the average values of $n$ for the biased and unbiased samples respectively, each with their standard deviations. The fourth and fifth items show the average values of $n$ and standard deviations for the thick samples $(d \geq 50 \mathrm{~nm})$ and the thin samples $(d<50 \mathrm{~nm})$, respectively.

The fit values of $n$ over the energy range of 1.2 to $6.5 \mathrm{eV}$ for seven of our samples and two from other researchers are displayed in Figure 4. In the legend of the graph in Figure 4, we have listed the thickness to which each sample was sputtered and the bias voltage at which it was sputtered. (A note on sample number is also apropos: the number is the (2-digit) year), the (2-digit) month, and the (2-digit) day that the sample was deposited.) Our samples and Liddell's ${ }^{13}$ mostly lie within a narrow band. All samples show normal dispersion over the range and the general shapes of the different fits of our samples' $n$ are essentially the same up until about $5.5 \mathrm{eV}$. Above about $5.5 \mathrm{eV}$, some curves climb more steeply than others. This is because the absorption band edge fitted for same samples is lower than for others; 
though for all our samples, it will be seen to be relatively high. The index is naturally dispersive below an absorption resonance. Data extracted from Figure 7 of Mahmoud ${ }^{14}$ do not correlate with others. Mahmoud's films are significantly more dispersive than others. The refractive index derived for Mahmoud's films, which were prepared by sol gel on hot glass, increases rapidly above $3 \mathrm{eV}$. This correlates with the band edge of his material at $3.82 \mathrm{eV} \cdot{ }^{14}$ His material is different than thin films prepared by others, perhaps because of impurities in his material which could have diffused out of the glass. ${ }^{1}$

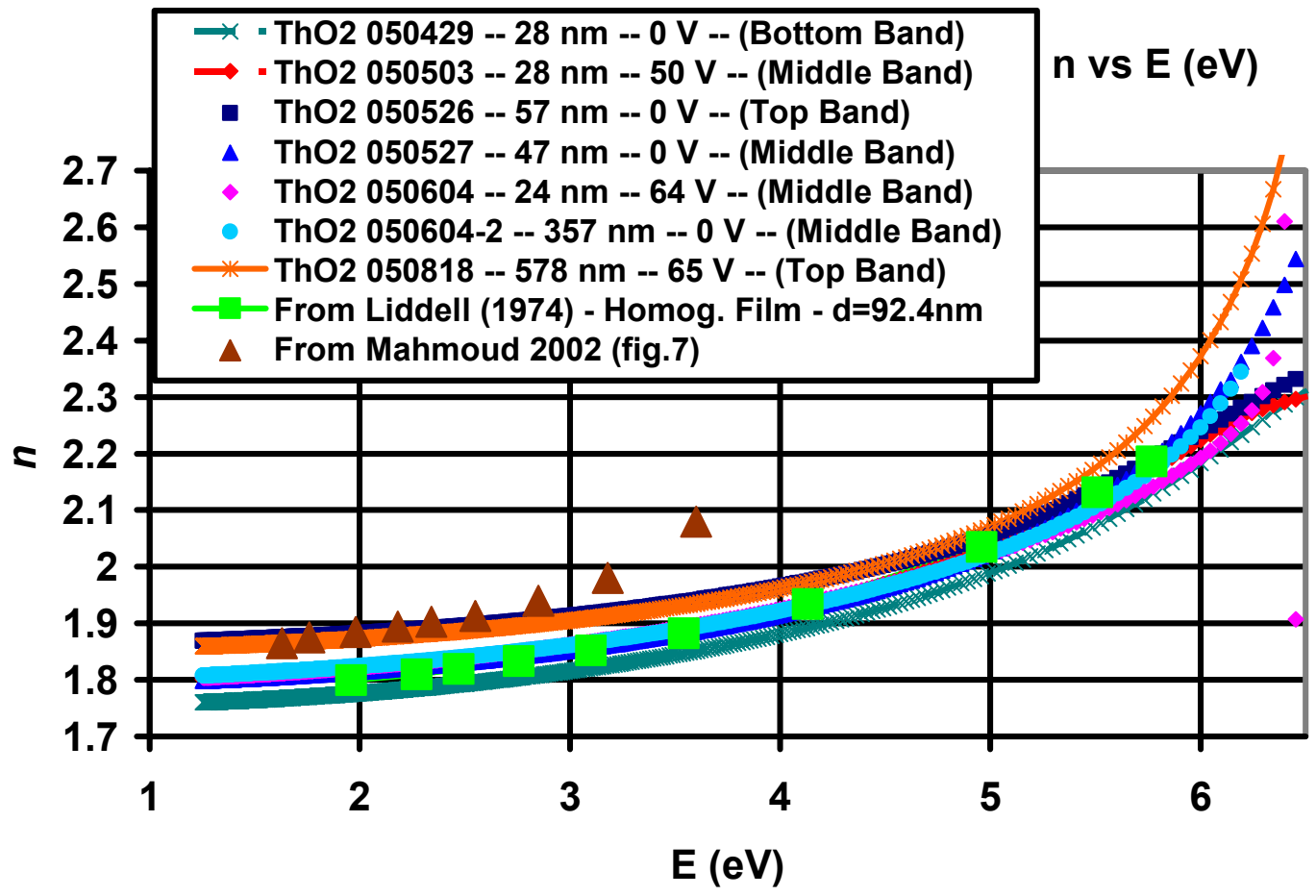

Figure 4: Reported values of the index of refraction for seven samples. The legend shows thickness as measured by ellipsometry, and the bias voltage at which each sample was sputtered. The legend labels each of the seven samples by the sample thickness as measured by ellipsometry, the bias voltage at which it was sputtered, and the band into which the sample falls. The values of $n$ reported by Heather Liddell (1974) and as derived from fig. 7 of Mahmoud (2002) are also included for comparison. ${ }^{13,14}$

\subsection{Absorption $\alpha$ and band gap.}

We turn our attention to the absorption properties of $\mathrm{ThO}_{2}$ thin films. Our goal was to obtain both the index of refraction and the $k$ of thoria. We observed that for the ellipsometric data between 1.2 and $6.5 \mathrm{eV}$, the fitting of $n$ and the fitting of $k$ could be conducted relatively independently for materials which are largely transparent in the visible and near UV. We proceeded in the following way:

1. The first step was to fit the measured ellipsometric $\Psi$ and $\Delta$ values for the thin film deposited on silicon, to obtain $n$, assuming no $k$.

2. Then $k$ is fit to the transmission data obtained from the samples on quartz, using the obtained values of $n$, first(2a), point-by-point, and then (2b) using a Tauc-Lorentz oscillator model.

3. The second fit on silicon was a fit of $n$ using the values of $k$ we obtained from the transmission data.

We found that between the two fits on the same silicon sample with the addition of absorption, the values of $n$ that we obtained did not change significantly. This is shown schematically in Figure 5. 
We note that adding $k$ between the first and second fits on silicon did not change the values of $n$ significantly. Therefore, it appears that the values of $n$ are not greatly dependant on the presence of $k$, when $k$ is small. These indications give us confidence that our results for each of $n$ and $k$ are mutually independent. This makes it easier to determine thicknesses. From $k, \alpha(\alpha=4 \pi k / \lambda)$ is calculated and from it $\alpha d$.

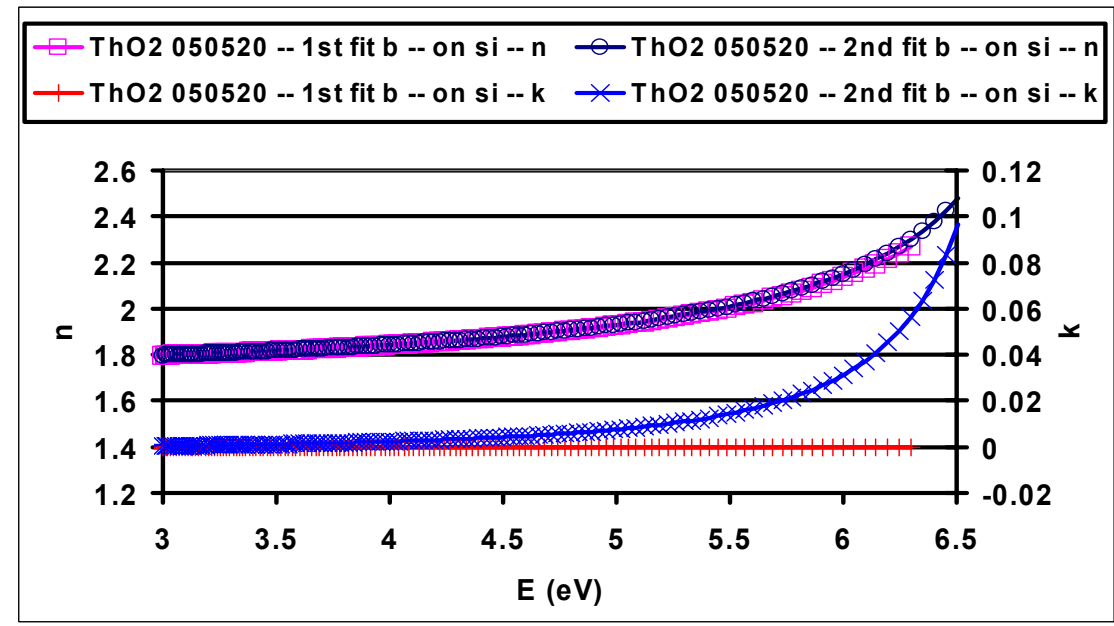

Figure 5: Plots showing $n$ and $k$ for two different fits on the same sample. The first fit of $n$ was done assuming no absorption. The second fit of $n$ was done after fitting the absorption. Note how $n$ did not change much after adding absorption.

\section{alpha*d vs E}

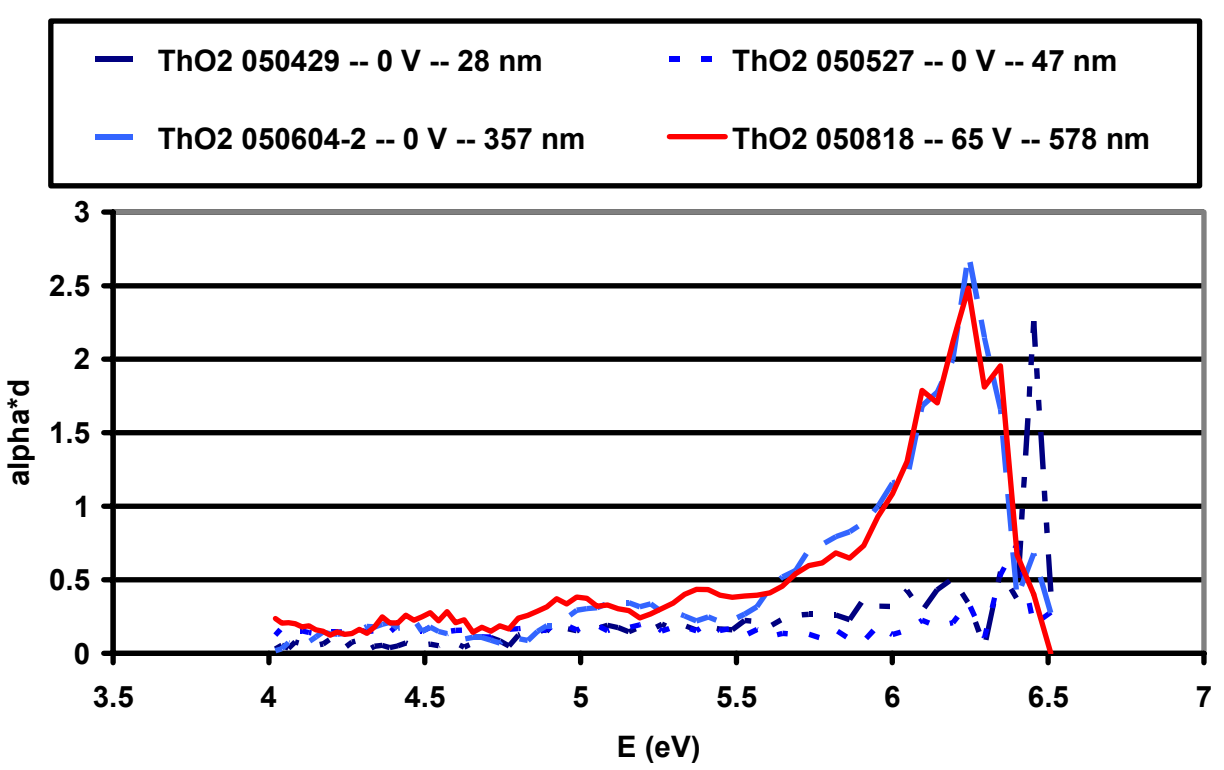

Figure 6: Plots showing $\alpha d$ vs. $E$ on four samples. Here $\alpha$ (from $k$ ) was fit point-by-point.

In Figure 6, we plot $\alpha d$ against photon energy for four samples. We used $\alpha$ d rather than just $\alpha$ as the ordinate, because we wanted to know if any features we might detected in $\alpha$ data were significant. Transmission is proportional as $e^{-\alpha d}$, where $d$ is the thickness of the sample, so a value of $\alpha \mathrm{d}>0.1$ could be significant, since this will produce an attenuation of transmission of $10 \%$. This difference can readily be detected. The value of $\alpha$ is calculated from point-by-point fits of $k$ to provide the data for Figure 6. The uncertainty in the data increases at higher energies because of the sources' poor UV output at the time these measurements were made. 


\section{DISCUSSION}

Analysis shows that the dramatic increase in absorption at about $6 \mathrm{eV}$ appears to be the onset of the band edge. Note that there is not a great deal of difference in the absorption of the biased samples and the unbiased samples in spite of thickness differences. The "waves" in the curves at low energy for the thick samples might be either absorption features or interference fringes which the fitting program was not entirely able to remove. These are gone after the subsequent step, where we modeled $k$ in the band edge region with one or more Tauc-Lorentz (T-L) oscillators. But in these plots we do not use the values the models produce since that would obscure the physics.

We are interested in the location of the band gap of thin film $\mathrm{ThO}_{2}$. For a detailed discussion of band gaps in semiconductors, see ref. 15. From our calculated values of $\alpha$, we plotted $\alpha^{2}$ (and $\alpha^{1 / 2}$ ) vs. $E$ to find the direct (and a

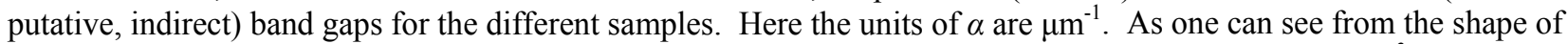
$\alpha d$ vs. $E$ in Figure 6, there is only a narrow section of the complete graph that we can use to extrapolate $\alpha^{2}$ to 0 .

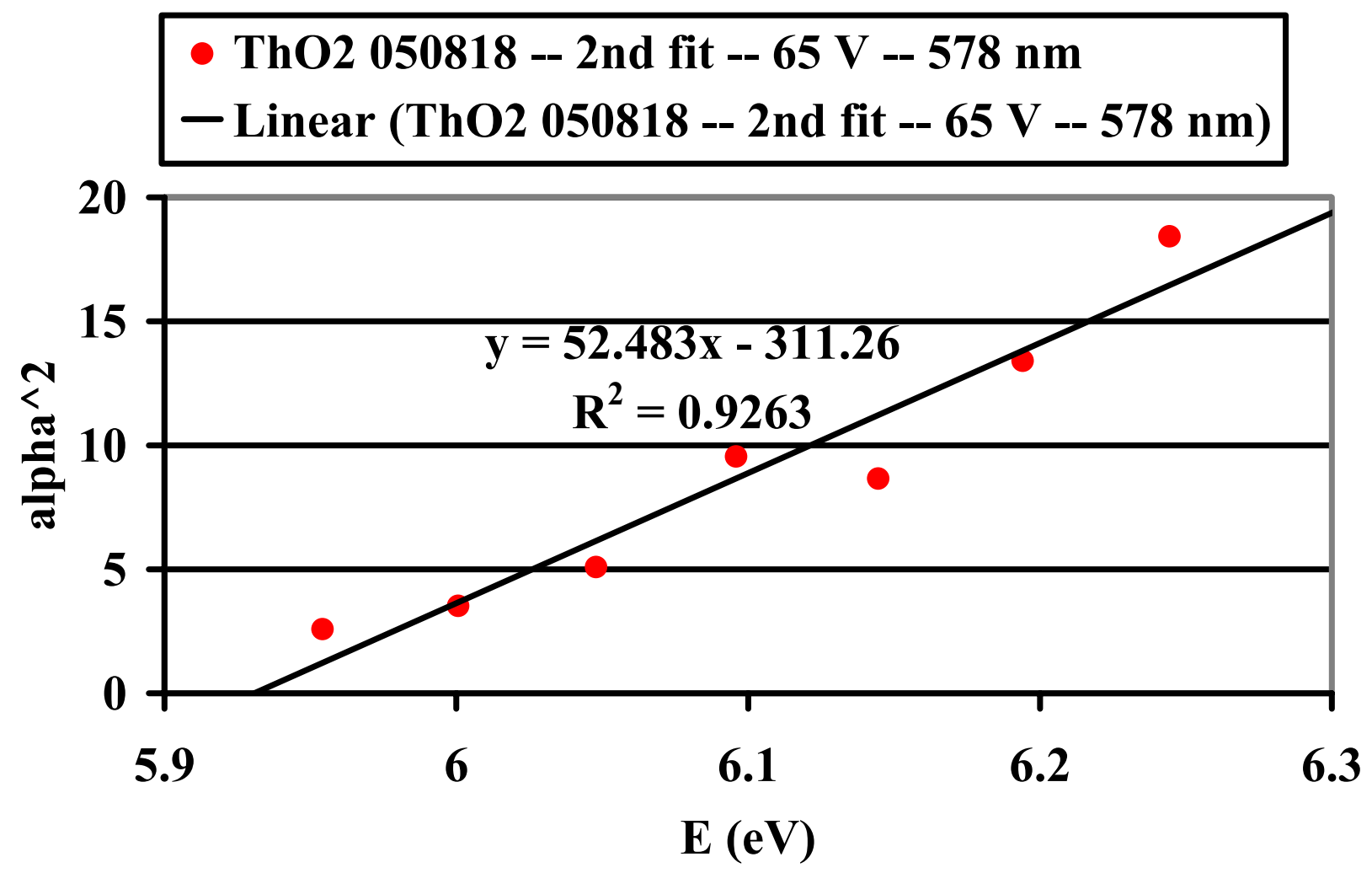

Figure 7: A plot of $\alpha^{2}$ vs. $E$ for sample ThO2 050818 , sputtered at $65 \mathrm{~V}$ bias voltage to a thickness of $539 \mathrm{~nm}$, with a linear fit. This fit extrapolates to $\alpha^{2}=0$ at $E=5.9 \mathrm{eV}$ and $\alpha$ has units of $\mu \mathrm{m}^{-1}$.

In Figure 7, we see a representative linear fit to a plot of $\alpha^{2}$ vs. $E$ for a sample (ThO2 050818). In this case, the fit extrapolates to $\alpha^{2}=0$ at $E=5.9 \mathrm{eV}$. For each of our samples in plotting $\alpha^{2}$ vs. $E$, the extrapolated band gap energy gave an average of $E_{g}=6.1 \pm 0.15 \mathrm{eV}$. We have modeled all of our data and provide the values elsewhere 2 .

Does thoria also exhibit an indirect gap? Many of our samples also exhibited some evidence of absorption below the band gap which might be due to states in the gap or evidence of an indirect absorption edge. It is important to proceed carefully here. The T-L model was devised for amorphous semiconductors. Amorphous semiconductors can have extensive absorption in the gap, and the models fit (below band gap) $k$ to give absorption parameters in the T-L model. 
What we have here is a crystalline dielectric. In any case we use point-by-point values of $k$ to calculate plotted $\alpha^{1 / 2}$ vs. $E$ to see if the sub band-gap absorption is compatible with an indirect band gap. We again restricted our domain to only use the range over which $\alpha^{1 / 2}$ was increasing approximately linearly with increasing $E$.

For materials like Si and Ge, the indirect band gap appears for $\alpha$ less than $0.01 \mu \mathrm{m}^{-1} .{ }^{15}$ If the material were like these, one would need a $>10 \mu \mathrm{m}$ film to provide robust evidence for an indirect band gap. None of our samples were sufficiently thick to show this low of absorption. But for completeness and because materials which have ionicity could exhibit indirect absorption higher than covalent silicon, we fit absorption data. Figure 8 shows a representative fit for an indirect band gap. This linear fit was done on the data from sample ThO2 050604, and extrapolates to $\alpha^{1 / 2}=0$ at $E_{i}=$ $2.6 \mathrm{eV}$. Most of our samples showed similar behavior, but there is no agreement as to what the indirect $E_{i}$ would be. Some even show $E_{i}<0$. We suggest instead that the absorption might be due to defects in the material. ${ }^{6-8}$

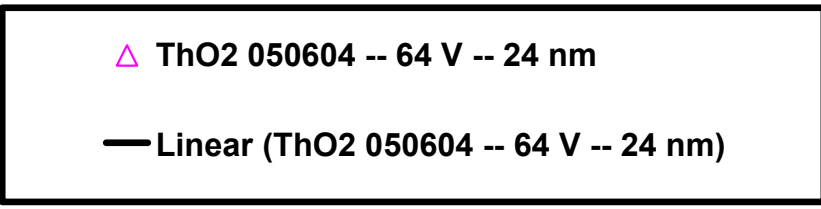

\section{alpha^(1/2) vs $E$}

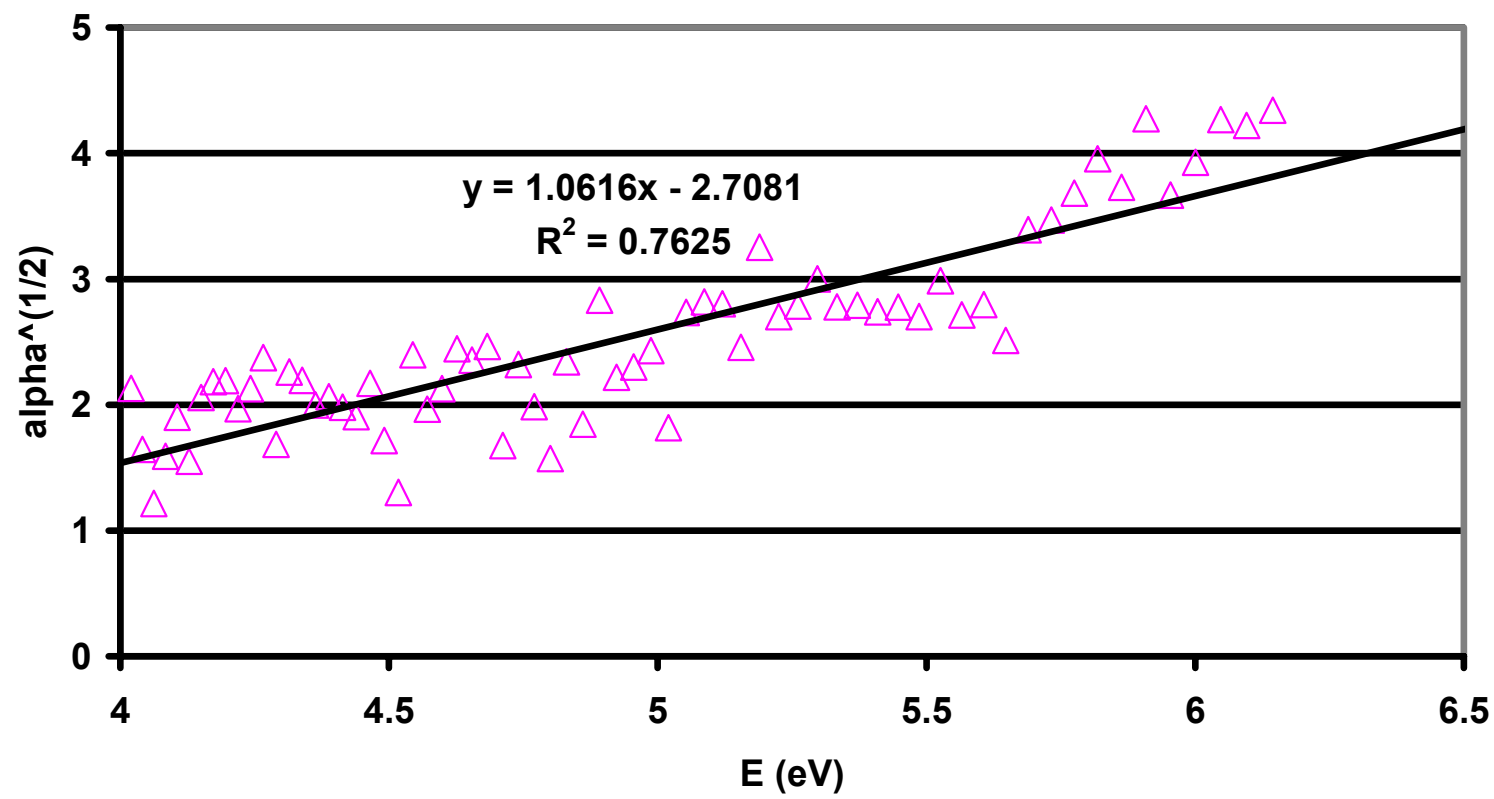

Figure 8: A plot of $\alpha^{1 / 2}$ vs. $E$ for sample ThO2 050604, sputtered at $-64 \mathrm{~V}$ bias voltage to a thickness of $6.589 \mathrm{~nm}$, with a linear fit. This fit extrapolates to $\alpha^{1 / 2}=0$ at $E=2.6 \mathrm{eV}$. Here $\alpha$ has units of $\mu \mathrm{m}^{-1}$.

Values for the direct band gap which have been reported for $\mathrm{ThO}_{2}$ range from $3.84 \mathrm{eV}^{14}$ to $5.92 \mathrm{eV}$ (ref. 1 and present report). The one computational study we are aware of places the gap at $4.5 \mathrm{eV} .^{5}$ The values which have been obtained and preparation details are summarized in Table II.

How could a crystalline material like $\mathrm{ThO}_{2}$ have such a wide range ( 3.8 to $6.0 \mathrm{eV}$ ) of apparent direct band gaps? Several comments are important. First, samples mentioned in the table are both bulk and thin films. The figure of $4.1 \mathrm{eV}$ quoted in the table for crystals are not the band gap but the transparency (absorption) edge. It is not possible to measure the band gap, which shows itself most clearly when $\alpha$ approaches $10^{4} \mathrm{~cm}^{-1}(\mathrm{k}>\sim 0.017$ at these energies) using the transmission of bulk samples when subband gap absorption features are present. A small amount of absorption could easily move the absorption edge to lower energies in bulk samples. The advantage of thin films is that absorption in the range expected for direct edges can be measured. Both Sviridova \& Suikovskaya ${ }^{3}$ and we have seen evidence for absorption below the band edge, and we can also clearly see the direct band edge. 
Table II: Selected reports on the absorption edge or band gap (BG) of $\mathrm{ThO}_{2} \mathrm{eV}$.

\begin{tabular}{|l|l|l|l|l|}
\hline Author & Ref. \# & BG (eV) & Preparation Method & Substrate \\
\hline Mahmoud & 14 & 3.84 & Spray pyrolysis T $\geq 300 \mathrm{C}$ & Glass \\
\hline Rivas-Silva & 5 & 4.6 & Computation (REX) & \\
\hline Neeley et al & 16 & 4.1 & Crystals & Bulk \\
\hline Rodine \& Land & 7 & 5.75 & Flux-grown Crystal & Bulk \\
\hline Griffiths & 8 & 5.8 & Arc-fusion Crystals & Bulk \\
\hline Sviridova \& Suikovskaya & 3 & 5.9 & Spin-on+Bake@ T $\geq 400 \mathrm{C}$ & Fused Quartz \\
\hline Evans \& Allred (Average) & & 5.92 & Reactive Sputtering & Fused Quartz \\
\hline
\end{tabular}

Second, while Sviridova \& Suikovskaya do not provide an estimate of the band gap of their material, they do tabulate the samples' thicknesses and transmission at 4 or 5 wavelengths near where the samples go from mostly transparent to mostly opaque. ${ }^{3}$ From this we calculated $\alpha d$ and then plotted it to calculate the band gap. This was remarkably constant at $\sim 5.92 \mathrm{eV}$ for both samples prepared using thorium chloride and those using thorium nitrate (See Fig. 9). We obtained values for the band gap in the same range.

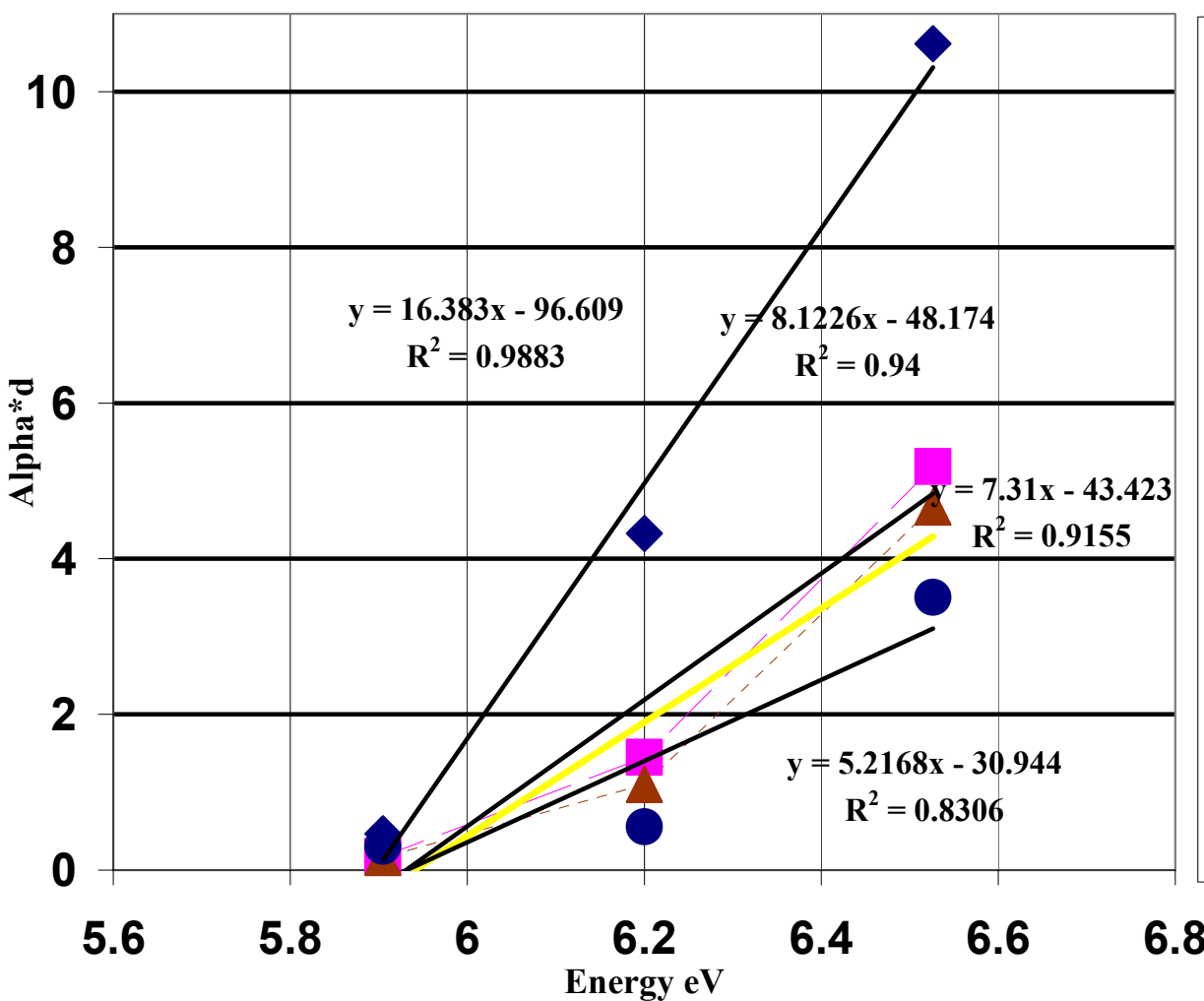

Figure 9: A plot of $\alpha^{2}$ vs. $E$ for solvent-prepared $\mathrm{ThO}_{2}$ films ${ }^{3}$ with a linear fit. These fits can be seen to extrapolate to $\alpha^{2}=$ 0 at $E=5.9 \mathrm{eV}$. Here $\alpha$ was extracted by us from Sviridova, and. Suikovskaya transmission data. The legend on the left gives the temperatures which the solution coated substrates were heated. The solution was thorium nitrate in ethanol. The linear regression equations and lines from upper left to lower right belong to temperatures 300 to $700 \mathrm{C}$.

Both our samples and reference 3 were deposited on very inert substrates; whereas Mahmoud used glass of unspecified composition. ${ }^{14}$ Standard glasses include mobile ions (especially alkali metals) which can diffuse into heated thin films, 
altering their optical and electronic properties. In the case of silica-based glasses, all have a narrower transparency range than pure silica and it is possible that this is true for thoria deposited on hot glass. Unfortunately no compositional analysis of his material is available in the literature. In any case, it is evident that whatever Mahmoud's material is, the band gap of his material is $<4 \mathrm{eV}$ as his careful measurements and calculations show. On the other hand, we can conclude that the band gap of pure thoria is probably just below $6 \mathrm{eV}$ since this is the consensus of those who have used pure materials, prepared on inert surfaces, and measured carefully. ${ }^{3,7,8, \text { and } 13}$

A second issue: One notes in figure 7 that the absorption drops precipitously after reaching a maximum at about $6.2 \mathrm{eV}$ for the two thickest samples. The absorption band appears to be narrow (the full width at half max is about $0.4 \mathrm{eV}$ ). This is consistent with the hypothesis that the upper band is built up mainly of states from the empty $5 \mathrm{f}$ levels of thorium (+4). This could produce a band which was narrow due to low overlap of f orbitals on different atoms. But does the material actually become more transparent above $6.2 \mathrm{eV}$ ? To test this we fit using a standard optical multilayer model the $10.2 \mathrm{eV}$ angle-dependent reflectance of three $\mathrm{ThO}_{2}$ thin film samples: the two thickest and one thinner, 48 $\mathrm{nm}$. The average \pm std dev calculated $n, k$ and $\alpha$ values were respectively, $0.87 \pm 0.12,0.48 \pm 0.05,47.3 \pm 5.5 \mu \mathrm{m}^{-1}$. The data and the fit to the thickest sample are shown in Figure 10. The model did not allow us to model with roughness on top. We note that the fit at low angles is deficient. The alpha is almost a factor of 10 higher at $10.2 \mathrm{eV}$ than at $6.2 \mathrm{eV}$. The question of whether there is relative greater transparency between 6.2 and $10.2 \mathrm{eV}$ is still open.
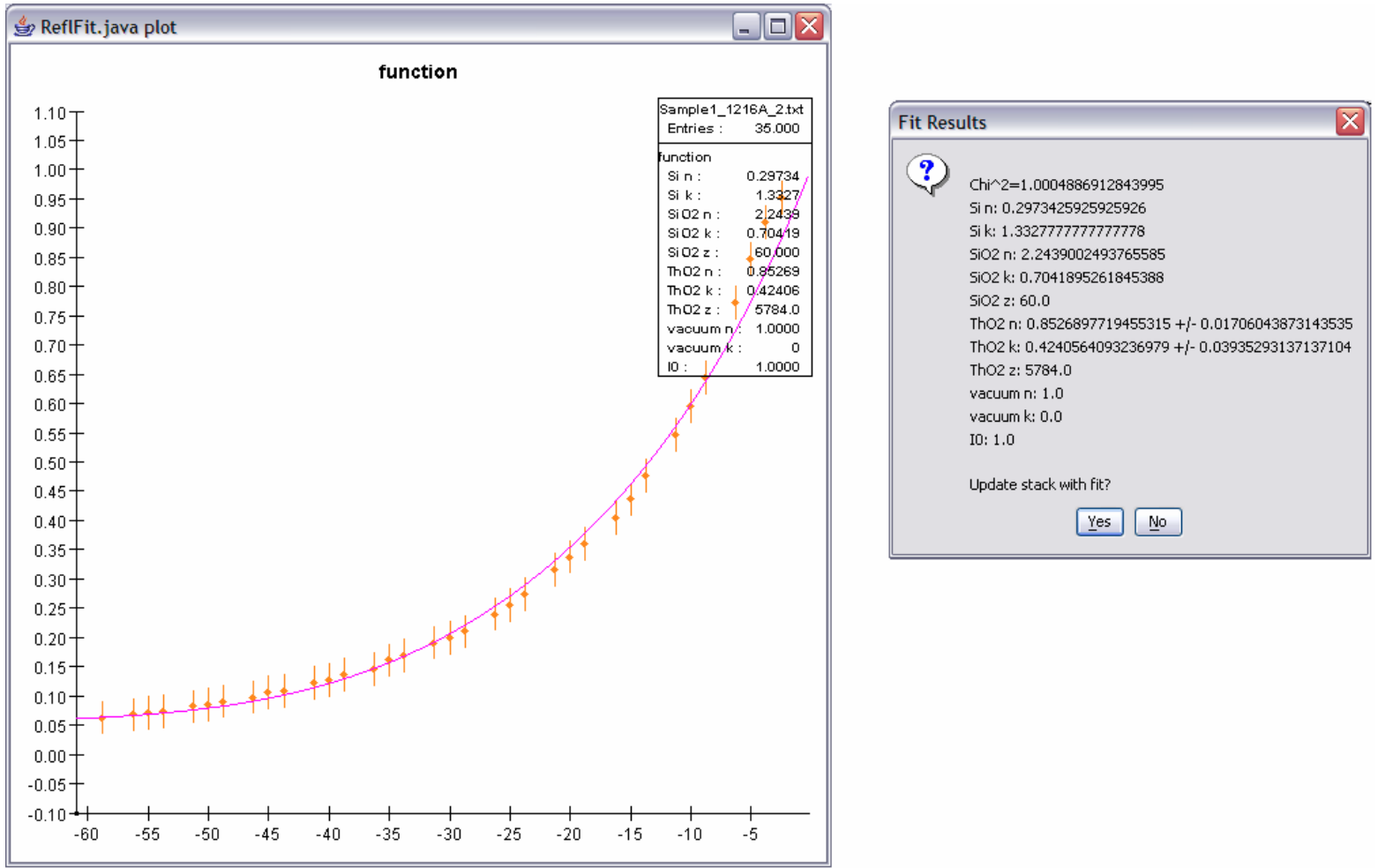

Figure 10: A fit of $n$ and $k$ for the data at $10.2 \mathrm{eV}$.

\section{CONCLUSIONS}

The direct band gap of $\mathrm{ThO}_{2}$ is about $5.9 \mathrm{eV}$. We find no evidence of an indirect gap. Spectroscopic ellipsometry can be used to determine characteristics useful for EUV research, for example the thickness of thin $\mathrm{ThO}_{2}$ films and that the material is probably not fully dense. DC-biased sputtering cannot be reliably expected to alter the indices of refraction of $\mathrm{ThO}_{2}$ and thus the density of thin films deposited by RF magnetron sputtering. ${ }^{1}$ Substrate heating or RF-biased RF magnetron sputtering might solve some of these problems. 


\section{ACKNOWLEDGEMENTS}

A BYU ORCA Scholarship to William Evans and BYU Department of Physics and Astronomy research funds aided in the research. We also acknowledge gratefully the financial contributions of V. Dean and Alice J. Allred and Marathon Oil Company for gifts to Brigham Young University for thin-film research. We thank all the members of the BYU EUV team for their support, especially Amy Baker for XPS, Jed Johnson for advice in sputtering films, Tom Dahlquist and Trevor Jensen for monochromator repairs, Nichol F. Brimhall for help with data fitting, and John E. Ellsworth and Robert Lawton for electronic and vacuum support.

\section{REFERENCES}

1. W.R. Evans and D.D. Allred, "Determining Indices of Refraction for $\mathrm{ThO}_{2}$ Thin Films Sputtered Under Different Bias Voltages from 1.2 to $6.5 \mathrm{eV}$ By Spectroscopic Ellipsometry" Thin Solid Films, In Press.

2. W.R. Evans, Honors Thesis, Determining Optical Constants for ThO $\mathrm{O}_{2}$ Thin Films Sputtered under Different Bias Voltages from 1.2 to $6.5 \mathrm{eV}$ by Spectroscopic Ellipsometry, (BYU Dec. 2005), available at http://www.physics.byu.edu/faculty/allred/cv/Theses/WRE/Bill's\%20ThO2\%20Ellipsometry\%20Thesis\%20-$\% 2017 \% 20$ Oct $\% 202005$.pdf

3. A. I. Sviridova and N. V. Suikovskaya. "Transparency limits of interference films of hafnium and thorium oxides in the ultraviolet region of the spectrum" Optics and Spectroscopy, 22, 509-512, 1965.

4. R. C. Linares, "Growth and properties of $\mathrm{CeO}_{2}$ and $\mathrm{ThO}_{2}$ single crystals," Journal of Physics, Chemical Solids, 28, 1285-91, 1967.

5. J. F. Rivas-Silva, J. S. Durand-Niconoff, M. Berrondo, "Electronic calculations on fluorides and oxides of Zr, Hf and Th," Computational Materials Sci., 18, 193-198, 2000.

6. See, for example. T. R. Griffiths, and James Dixon, "Optical Absorption Spectroscopy of Thorium Dioxide: Analysis of Oxidizing and Reducing Anneals and Gamma and UV Radiation on both Flux-grown and Arc-fused Single Crystals." J. of Chemical Society, Faraday Transactions, 88, 1149-1160, 1992, and ref. cited within, especially ref. 2-9.

7. Elward T. Rodine and Peter L. Land, "Electronic Defect Structure of Single-Crystal $\mathrm{ThO}_{2}$ by Thermoluminescence" in Phys Rev. B 4, 2701-2724, 1971 and ref. cited within. Also PhD dissertation: Elward Theodore Rodine, Thermoluminescence of Thorium-Oxide Single Crystals, University of Nebraska-Lincoln 1970; at http://digitalcommons.unl.edu/dissertations/AAI7103654/

8. Trevor Griffiths, and James Dixon, "Electron irradiation of single crystal thorium dioxide and electron transfer reactions," Inorganica Chimica Acta, 300-302, 305-313, 2000.

9. Jed E. Johnson, David D. Allred, R. Steven Turley, William R. Evans, and Richard L. Sandberg, in ActinidesBasic Science, Applications, and Technology, 893 (Material Research Society Symposium Proceedings, 2005), Sarrao, A. Schwartz, M. Antonio, P. Burns, R. Haire, H. Nitsche, eds., 207-214, 2005.

10. Nicole F. Brimhall, Amy Baker, R. Steven Turley, and David D. Allred, "Thorium-based mirrors in the extreme ultraviolet," 6317-36, in Advances In X-Ray/EUV Optics, Components, And Applications, Ali M. Khounsary, and Christian Morawe, eds. Proceedings SPIE, 6317, pages not available, 2006.

11. L. J. Bissell, D. D. Allred, R. S. Turley, W. R. Evans, J. E. Johnson, "Determining Ruthenium's Optical Constants in the Spectral Range 11-14 nm," in Optical Constants of Materials for UV to X-Ray Wavelengths, edited by Regina Soufli, and John F. Seely, Proceedings of SPIE, 5538, ) 84-91, SPIE, Bellingham, WA, 2004.

12. Powder Diffraction File, (Joint Committee on Powder Diffraction Standards), Inorganic, Sets 1-5, 527. Index number 4-0556, Philadelphia, PA, 1960.

13. H. M. Liddell, "Theoretical Determination of the Optical Constants of Weakly Absorbing Thin Films" J. Phys. D: Appl. Phys., 7, 1588-1596, 1974.

14. S. A. Mahmoud, "Characterization of Thorium Dioxide Thin Films Prepared by the Spray Pyrolysis Technique" in Solid State Sciences, 4, 221-228, 2002.

15. M. Fox, Optical Properties of Solids. pp. 54-55, 58-59, 63-65, Oxford University Press, Oxford, UK, 2001.

16. V. I. Neeley, J. B. Grubert, and W. J. Gray, "F Centers in Thorium Oxide," Physical Review, 158, 809-813, 1967. 